\title{
CREATIVIDAD COMO HERRAMIENTA SOCIOEDUCATIVA. ACTIVIDAD PILOTO "MEDIOAMBIENTE, SOCIEDAD Y BARRO"
}

\author{
María del Palacio Pérez \\ Educadora Social \\ mariadelpalacio97@gmail.com \\ Ana Isabel Isidro de Pedro \\ Universidad de Salamanca \\ anyis@usal.es
}

Recepción Artículo: 6 febrero 2020 Admisión Evaluación: 4 marzo 2020

Informe Evaluador 1: 1 enero 2020

Informe Evaluador 2: 1 enero 2020

Aprobación Publicación: 20 abril 2020

\section{RESUMEN}

El presente trabajo versa sobre la ejecución de la Actividad piloto "Medioambiente, sociedad y barro", una propuesta de intervención socioeducativa a través del arte. La actividad piloto, de temática medioambiental y transformadora, se realiza a través del barro, con un grupo de menores pertenecientes a un centro de socialización y adultos aficionados al barro, y en relación a los datos de la fundamentación teórica, que abarcan diversos conceptos como autoestima, tolerancia, transformación individual y social y actitud crítica como aspectos posibles a desarrollar a través de la creatividad. En su desarrollo, se presentan dos tipos de baldosas de arcilla: unas intactas, en las que se representa un tema a partir de láminas ilustrativas sobre la problemática socioambiental; y otras dañadas previamente, que habrán de reconstruir. De este modo, se permite la reflexión sobre los problemas ambientales y la relación entre los problemas naturales y sociales, así como entre el conflicto de inserción y el conflicto medioambiental, pudiendo abarcar temas como la sociedad de consumo o el sistema educativo tradicional y los "deshechos" que generan. El resultado es un mural que muestra la naturaleza dañada en la mitad de sus piezas y la transformación de lo dañado en la otra mitad.

Palabras clave: arte; creatividad; medioambiente; potencial socioeducativo; transformación individual y social

\section{ABSTRACT}

Creativity as a socio-educational tool. Pilot activity "Environment, society and mud". The present work is about the execution of the pilot activity "Environment, society and mud", a socio-educational intervention proposal through art. The pilot activity, of environmental and transformative thematic, is carried out through mud, with a group composed by minors belonging to a socialization center as well as adult amateur pot- 
ters, in relation to the theoretical foundation data, which include self-esteem, tolerance, individual and social transformation and critical attitude as possible aspects of developing through creativity. During its development, two types of clay tiles are presented: intact ones, in which a theme is represented from illustrative sheets about the socio-environmental problem; and others previously damaged, which will have to be rebuilt. In this way, reflect on environmental problems and the relationship between natural and social problems are allowed, as well as between the insertion conflict and the environmental conflict, being able to cover topics such as the consumer society or the traditional education system and the "waste" they generate. The result is a mural that shows the damaged nature in half of its pieces and the transformation of the damaged in the other half.

Keywords: art; creativity; environment; socio-educational potential; individual and social transformation

\section{ANTECEDENTES}

Este trabajo representa la oportunidad de ahondar en la comprensión de la creatividad como herramienta socioeducativa, desde una aproximación tanto teórica como práctica. Desde la Educación Social se trabaja con personas, seres complejos, cuyo desarrollo integral pasa también por el desarrollo de la creatividad, la cual han intentado definir numerosos autores.

En general, se puede observar que la creatividad es definida como una forma diferente de pensamiento (Romo, 1997) 0 de resolución de problemas (Martín González y Marín Viadel, 2006), donde destaca saber encontrar las dificultades y una disposición abierta y crítica (Guilford, s.f., citado en Romo, 2007) y formular "buenas" preguntas (Torrance y Myers, 1976; Trillas, 2018); cuyos resultados aportan novedad y valor (Romo, 1997); considerada una herramienta liberadora del pensamiento por De Bono, quien crea el concepto de "pensamiento lateral", definido como una actitud que permite tomar diferentes perspectivas y entender que cualquier forma de valorar es tan sólo una entre muchas posibles (1994, citado en Menchén, 2006); y como el elemento que ha estructurado el proceso de humanización (Romo, 2007); posible de desarrollar por cualquier persona (Romo, 1997); considerada como un "bien social" (Torre, 2006a, p. 585) y presente en cada una de las inteligencias múltiples de Gardner, relacionada, en el caso de la Inteligencia Interpersonal e Intrapersonal, con el autoconocimiento y autoestima (Solar Rodríguez, 2006); de carácter vital y cotidiano (Marina y Marina, 2013); interactivo y sistémico, que se da en la interacción entre las personas y el contexto sociocultural (Csikszentmihalyi, 1998, citado en Meller, 2019); con un gran potencial transformador tanto personal como social (Torre, 2006b), con numerosas aportaciones para la persona como: coraje, independencia de pensamiento y juicio, honestidad, perseverancia, curiosidad y voluntad para asumir riesgos (Torrance, 1986, citado en Sternberg, 1988, p. 68) o fortaleza mental y emocional e inconformismo (Amabile, 1989); e implicaciones educativas al definirla como "una actitud que debe ser trabajada porque desarrolla los procesos de pensamiento, estimula la sensibilidad hacia los problemas, ejercita las habilidades cognitivas y refuerza la voluntad para perseverar en la labor iniciada[... . (y relacionarla con) la formación integral de la persona y su integración efectiva en la sociedad" (Martín González y Marín Viadel, 2006, p. 56)

Resultaría de interés, y hasta necesario, abordar cómo desarrollar la creatividad, las diferentes propuestas y métodos en tal sentido; sin embargo, dadas las características de este trabajo, no es posible, quedando aquí explicitado para futuras ampliaciones.

Encontramos numerosas iniciativas socioeducativas en relación al desarrollo de la creatividad, como son: Ios talleres de salud mental del Hospital Psiquiátrico con el centro de arte Casal Solleric, en Mallorca, con objetivos como favorecer la socialización y autoestima de los participantes, entre otros (Reyes, 2018); dos proyectos de educación ambiental -"Arte Emboscado" y "Herbario sonoro" - de la Fundación Tormes (Salamanca), dada la necesidad detectada de llegar a más y más diversas personas (Fundación Tormes, 2019); desarrollo comunitario a través del arte en diferentes ciudades, que favorece la convivencia, participación y desarrollo económico, entre otros (ZOES, 2017; Renault, 2019); el proyecto Croqueta de Edelvives, donde la creatividad es una de sus seis claves, siendo el primer proyecto editorial basado en la neurociencia (Edelvives, 2019); Canarias ha sido pionera en hacer obligatoria en la etapa de primaria la asignatura Emocrea, para la educación emocional y la creatividad 
(Gimeno-Doñate, 2018); en varios institutos el neurocientífico Guillén trabaja educación emocional y creatividad, elementos que, necesariamente, se deben tratar conjuntamente, pues se complementan para lograr el pleno desarrollo de la persona (2019).

\section{OBJETIVOS DE LA ACTIVIDAD PILOTO}

La actividad piloto, de temática medioambiental y transformadora, busca:

0G1 Trabajar la creatividad en términos de resolución de problemas a través de la búsqueda de potencialidades a una lámina de barro dañada.

OG2 Trabajar la resiliencia y fortaleza emocional a través de la creatividad por medio de la reflexión y analogía entre los problemas ambientales, sociales y personales.

OG3 Favorecer la transformación individual y social a través de la creatividad, representada en la transformación del barro, potenciada por el trabajo en equipo y reflejada en la capacidad de cambiar la propia perspectiva sobre un problema medioambiental, social o personal.

\section{PARTICIPANTES}

Participan un grupo de menores en riesgo de exclusión social (alumnos de un centro de menores que no especificaremos para proteger su identidad), formado por 5 chicas y 2 chicos, de entre 11 y 17 años, elegidos por los educadores en función de su disponibilidad e interés, un joven más que se corresponde con el educador que les acompaña y un grupo voluntario de adultos -dos hombres y cuatro mujeres-, que cuentan con estudios universitarios como ADE o Trabajo Social y aficionados al barro; siendo así un encuentro entre infancia, juventud y edad adulta.

\section{METODOLOGÍA}

\section{Aspectos previos}

Para la organización y ejecución, se cuenta con la colaboración de Gerardo, profesor de barro. Una vez tomadas las decisiones pertinentes, se contacta con el centro de menores, para corroborar su aprobación. Se prepara una encuesta de satisfacción anónima para los participantes e imágenes de contenido socioambiental y, el día previo, el material y el espacio.

\section{Desarrollo de la experiencia}

Se encuentran delante de dos tipos de baldosas de arcilla, unas intactas y otras dañadas y rayadas previamente. En las intactas, se elige un tema a representar individualmente, utilizando la técnica del esgrafiado, a partir de las láminas ilustrativas sobre la problemática socioambiental recopiladas (ver llustración 1). De este modo, se permite la reflexión sobre un tema que tanto afecta a todos, pues los problemas ambientales no son sólo los problemas naturales, también sociales, ya que las desigualdades existentes en la sociedad derivan de las malas relaciones que ha mantenido ésta con la naturaleza en los últimos años, como se reconoce en la Conferencia de las Naciones Unidas sobre el Medio Humano celebrada en 1972 en Estocolmo (Calvo Roy y González de la Campa, s.f.) Por lo que el conflicto de inserción que viven los chicos se compara con el conflicto medioambiental. 
Ilustración 1. Algunos de los resultados de las baldosas esgrafiadas.

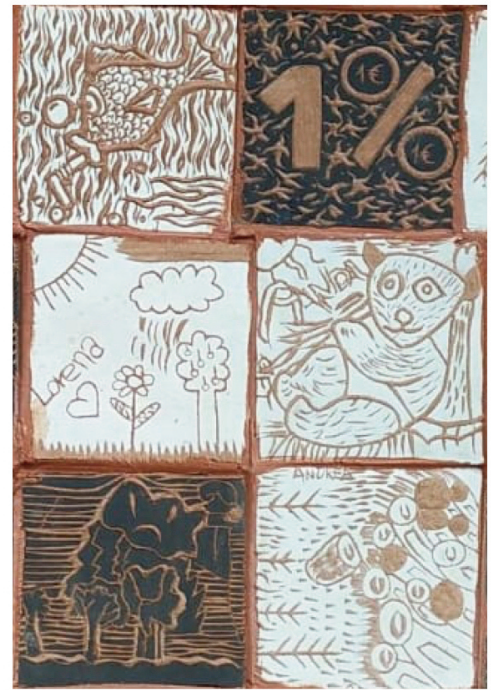

En las baldosas dañadas previamente, se refleja la necesidad de cambio y de transformación personal y social que requieren estos problemas socioambientales, representado por la transformación del barro que realizan los participantes para arreglarlas. También se reflexiona inevitablemente acerca de la sociedad del consumo, para la cual esta lámina de barro rota no sirve y sería desechada, al igual que las personas que no encajan en el sistema educativo tradicional tal como muestra Tonucci en sus ilustraciones y que Lorenzo Milani denominaba "Ios últimos" (Scuola de Barbiana, 2006); sin embargo, nosotros lo rescatamos y buscamos las fortalezas y potencialidades que tiene, porque todo y todos somos valiosos. En el proceso se hace referencia a los daños de la naturaleza, de la sociedad e incluso de sus propias vidas y se trabaja para la transformación de ello en algo nuevo, bonito, fuerte, útil, creativo (ver llustración 2, 3 y 4).

Ilustración 2. Rayones transformados en los tallos de unas plantas

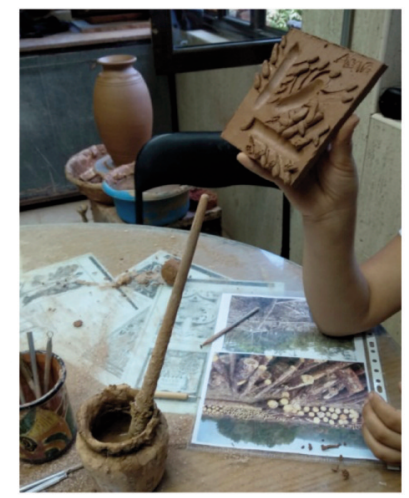


Ilustración 3.Muescas convertidas en las zanahorias de un conejo.

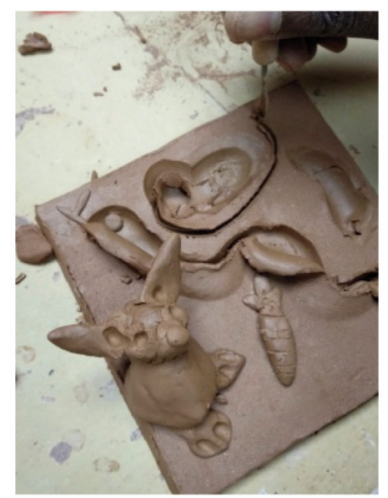

Ilustración 4. Baldosas agujereadas que, aprovechando el desperfecto, son transformadas en flores y pájaros.

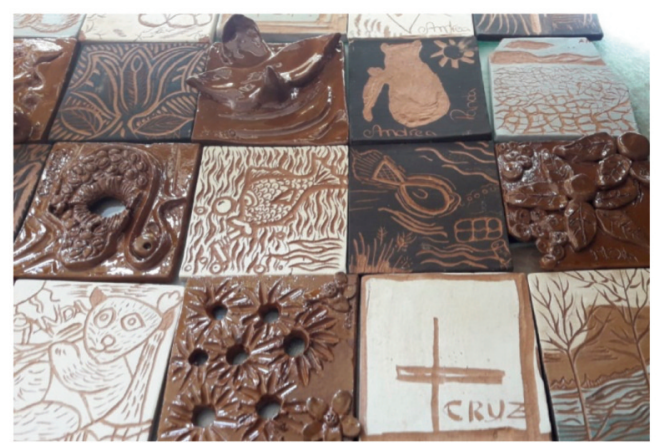

Durante el desarrollo, se atienden individualmente tanto las dudas técnicas como las necesidades que puedan surgir en relación a la motivación, inspiración, conflicto de opiniones, la necesidad de compartir ideas o de ser atendido. Así es como se va animando al diálogo, clave en esta actividad.

La duración del taller es de dos horas.

El resultado que se obtiene es un mural de unos 2x1,5 metros (ver Ilustración 5), que combina la representación de la naturaleza dañada en la mitad de sus piezas y la transformación de lo dañado, en la otra mitad. 
Ilustración 5. Colocación final del mural.

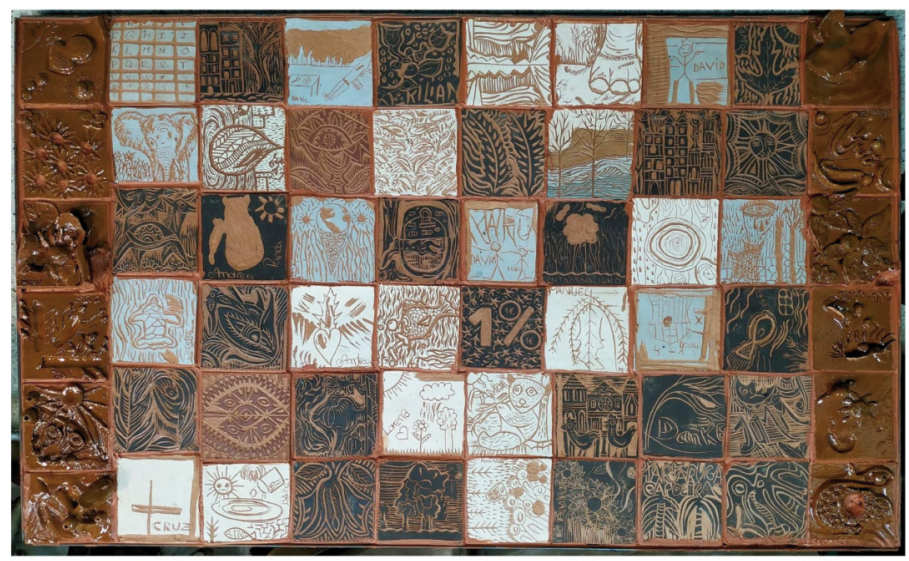

Tras cocer las piezas, el mural, que combina la representación de la naturaleza dañada y la transformación de lo dañado, ha sido colocado en el Centro de Educación Ambiental Irrecuperables Milani (Salamanca) (ver llustración 6).

Ilustración 6. Mural y panel descriptivo ubicados junto al recinto de aves irrecuperables en el CEA.

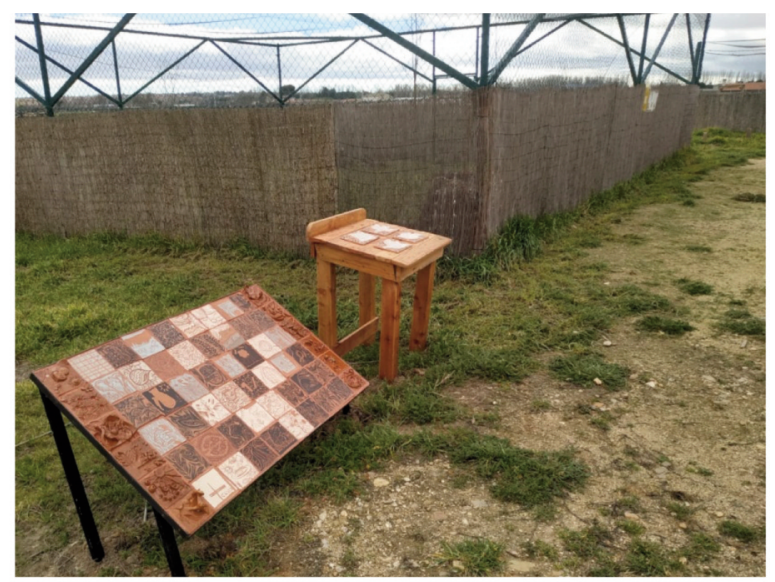

\section{Evaluación}

Se utiliza una metodología cualitativa, a través de notas de campo recogidas de la observación participante, y un cuestionario con escalas tipo Likert que permite conocer la perspectiva subjetiva de los sujetos y se anima a la redacción individual y voluntaria de una breve reflexión sobre sus expectativas, sentimientos, pensamientos, reflexiones y satisfacción. 


\section{ANÁLISIS Y DISCUSIÓN DE LOS RESULTADOS}

La información fue recogida en dos formatos: notas de campo y encuestas.

\section{Notas de campo}

Por un lado, tenemos las anotaciones de la hoja de observación que, por motivos de espacio, no se detalla, pero de la que se obtienen conclusiones clasificadas por momentos del desarrollo.

Se puede decir que la fase de esgrafiado ha sido útil a la hora de trabajar la motivación, la autoestima, la consciencia plena, la autoconfianza, la analogía entre problemas medioambientales y sociales, la transformación individual -desde los diferentes cambios de opinión-y social -desde la cooperación-. En esta fase nos encontrábamos con actitudes como miedo al error y a lo nuevo, motivación y autocrítica (que llevaba a repetir el trabajo hasta estar a su gusto), reflexiones propias sobre el medioambiente a partir de las imágenes, esfuerzo en transmitir todo lo posible mediante su baldosa, relajación y consciencia plena centrándose en el proceso, miedo e indecisión por estropear el trabajo, intentos de reforzar o corregir según el caso.

Por otro lado, en la fase de reconstrucción de la baldosa rota, se ha podido trabajar la búsqueda de soluciones y la resiliencia y fortaleza emocional desde el afrontamiento de los retos y la perseverancia, pues en ocasiones se podía apreciar frustración y desmotivación -"Esto no se puede arreglar (menor)", "¿Y yo qué hago con esto ahora? No sé qué hacer, ya no se me ocurre más (menor)"-, afirmaciones catastrofistas - "Los problemas no tienen solución (menor)"-, evitación de retos -"A mí fácil, fácil (menor)"-, aunque también búsqueda de retos difíciles, persistencia y astucia en la resolución.

Cabe destacar, que la mayor dificultad encontrada es el hecho de dirigir el taller y tomar notas; por ello, muchas son tomadas al finalizar y seguramente falte información. A pesar de ello, permite visualizar lo sucedido en cada fase de la actividad en su contexto.

\section{Encuestas}

Las encuestas están formuladas a modo de escala tipo Likert con cinco opciones: nada, poco, a medias, bastante y mucho. Además, en cada pregunta cuentan con un espacio para justificar su respuesta acompañado de una pregunta que ayude a ello. El análisis de dichos resultados fue realizado mediante el programa PSPP.

El primer ítem hace referencia al género de los participantes, como podemos observar, un $72,73 \%$ son mujeres y un $27,27 \%$ son hombres (ver Figura 1 ).

Figura 1. Diagrama de sectores por género.

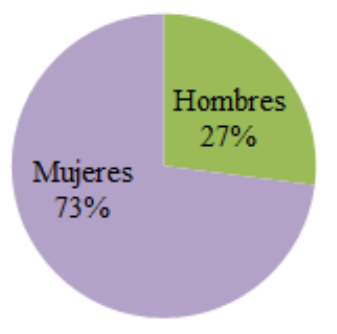


Tabla 1. Datos de edad

\begin{tabular}{|c|c|c|c|c|c|}
\hline EDAD & FRECUENCIA & PORCENTAJE & $\begin{array}{l}\text { PORCENTAJE } \\
\text { ACUMULADO }\end{array}$ & & \\
\hline 11 & 1 & $9,09 \%$ & $9,09 \%$ & & \\
\hline 13 & 1 & $9,09 \%$ & $18,18 \%$ & EDAD & \\
\hline 14 & 2 & $18,18 \%$ & $36,36 \%$ & Media & 31 \\
\hline 17 & 2 & $18,18 \%$ & $54,55 \%$ & $\begin{array}{l}\text { Desv. } \\
\text { Std }\end{array}$ & 21,9 \\
\hline 24 & 1 & $9,09 \%$ & $63,64 \%$ & Mínimo & 11 \\
\hline 51 & 1 & $9,09 \%$ & $72,73 \%$ & Máximo & 63 \\
\hline 58 & 1 & $9,09 \%$ & $81,82 \%$ & & \\
\hline 61 & 1 & $9,09 \%$ & $90,91 \%$ & & \\
\hline 63 & 1 & $9,09 \%$ & $100 \%$ & & \\
\hline TOTAL & 11 & $100,00 \%$ & & & \\
\hline
\end{tabular}

El segundo ítem atiende a la edad de los participantes (ver Tabla 1). Las edades con las que se ha trabajado van desde 11 hasta 63 años, un 54,55\% son menores, mientras que un 45,45\% son adultos, de los cuales, un $80 \%$ son mayores de 50 años.

Los ítems siguientes hacen referencia a las preguntas en cuestión.

En primer lugar, se analizan los resultados de las preguntas (P.) que evalúan satisfacción.

Sobre la satisfacción general, encontramos que todas las respuestas son positivas, con la máxima puntuación en el 82\% de respuestas tanto de la P. 1 (ver Figuras 2 y 3) como de la P. 8, referida al resultado final (ver Figuras 4 y 5). Algunas de estas justificaciones hacen referencia al buen ambiente y a que la experiencia es divertida, entretenida, interesante, creativa, enriquecedora y permite aprender, pensar y compartir. De los que respondieron (pues hay valores perdidos), les gustaría mucho $(55,56 \%)$ y bastante $(33,33 \%)$ repetir para aprender, compartir, conocer gente y disfrutar, y, en varias ocasiones, por el buen clima grupal generado.

Figura 2. P.1. ¿Te ha gustado la actividad?

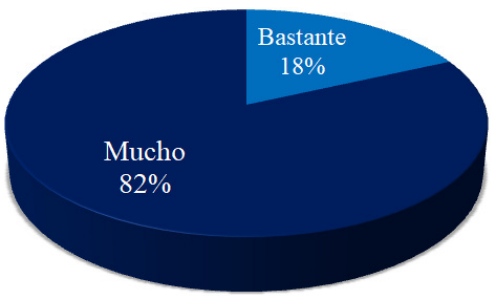


Figura 3. Resultados por edad P.1.

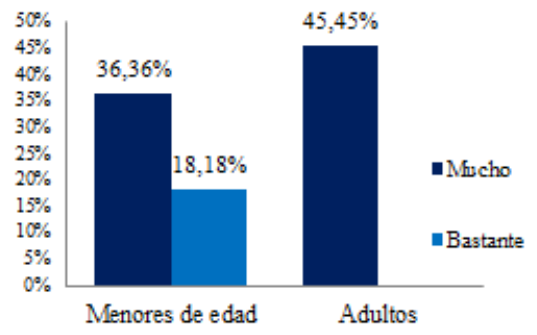

Figura 4. P.8. ¿Estás satisfecho con el resultado final?

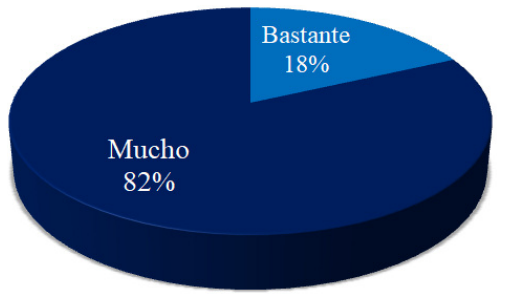

Figura 5. Resultados por edad P.8.

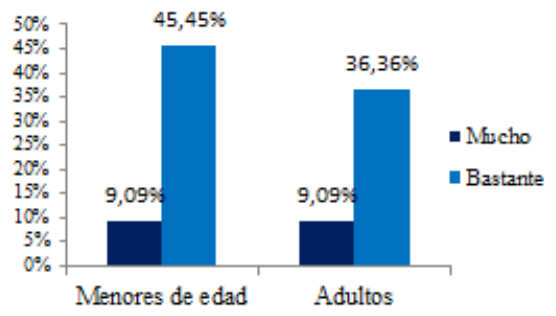

Respecto a la duración del taller, para la mayoría es adecuada, expresando algunas personas resultarle corta (ver Figuras 6 y 7 ).

Figura 6. P.9. ¿Crees que la duración del taller es adecuada?

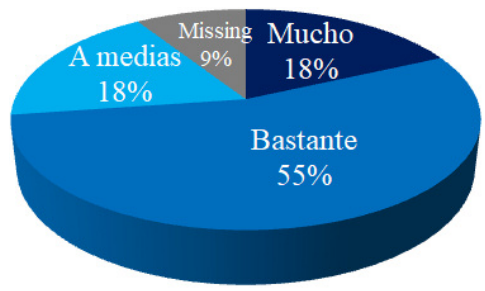


Figura 7. Resultados por edad P.9.

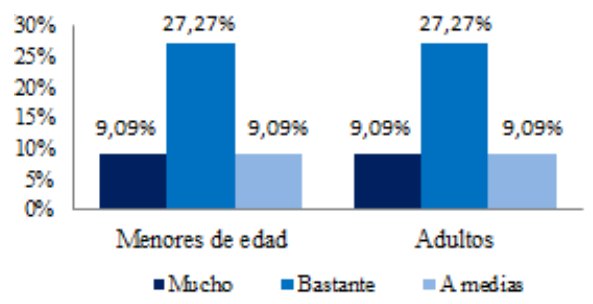

En relación al barro, la mayoría coincide en considerarlo el material adecuado para esa actividad, siendo los adultos el grupo más satisfecho con dicho material porque "es la propia materia de la Naturaleza, que se puede tratar bien o mal", es moldeable, expresivo y relajante (ver Figuras 8 y 9 ).

Figura 8. P. 10. ¿Crees que el barro es el material adecuado para esta actividad?

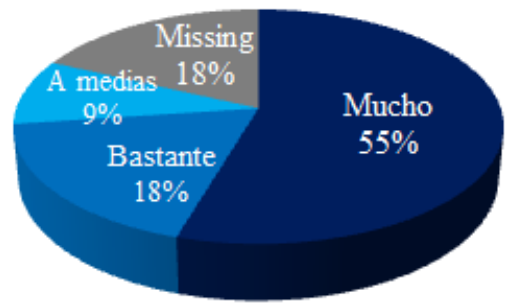

Figura 9. Resultados por edad P.10.

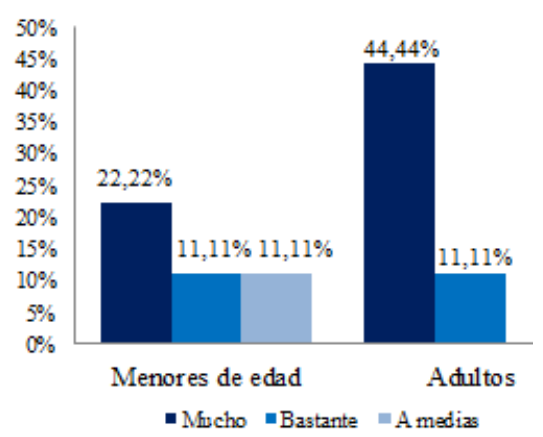

Finalmente, a la mayoría les gustaría mucho o bastante repetir la experiencia (ver Figuras 10 y 11). 
Figura 10. P. 11. ¿Te gustaría repetir la experiencia?

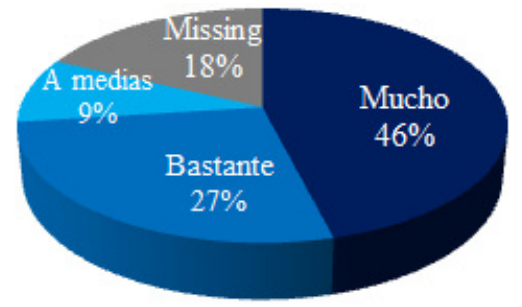

Figura 11. Resultados por edad P.11

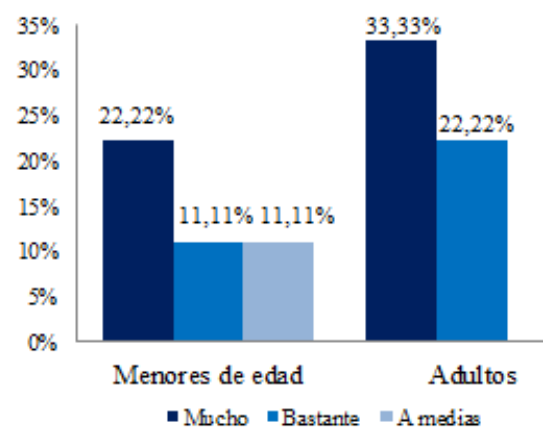

A continuación, se analiza el clima grupal y el ambiente generado durante la actividad.

Sobre el clima grupal encontramos gran satisfacción en todos los participantes, sin diferencias generacionales, con una puntuación media de 4,85 puntos sobre 5, siendo ésta la mayor puntuación alcanzada en la encuesta y una de las preguntas respondidas por todos los participantes (ver Figuras 12 y 13). De esto $-y$ de la referencia al buen ambiente en justificaciones de otras preguntas-, se deduce la importancia y el éxito de este factor en la realización de la actividad. Entre otros, se resalta la tranquilidad, la naturalidad y la cooperación grupal.

Figura 12. P. 2. ¿Has estado a gusto con el resto de personas del grupo?

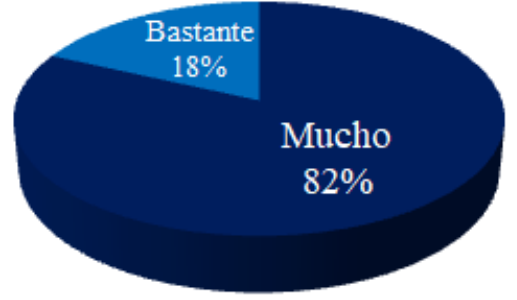


Figura 13. Resultados por edad P.2.

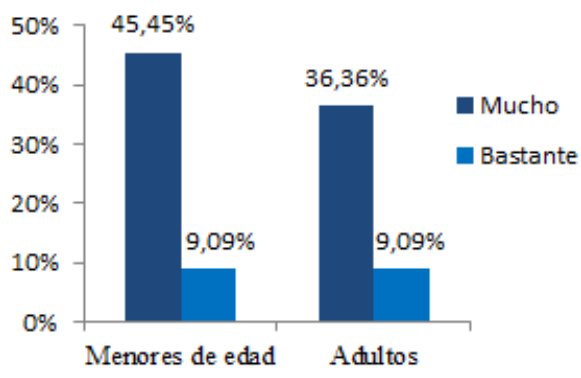

A continuación, se analizan las respuestas obtenidas en relación a la búsqueda de soluciones y potencialidades.

En esta cuestión hay diversidad de opiniones, predominando en un 80\% de los adultos una percepción de dificultad intermedia en cuanto a la búsqueda de soluciones para la baldosa rota, mientras que en los menores, un $50 \%$ de éstos lo consideraba de gran facilidad (ver Figuras 14 y 15). Algunas de las razones aportadas a la baja dificultad es tener mucha imaginación y para la alta dificultad se encuentran explicaciones como la incoherencia entre el resultado y la intención, el ser la primera vez en hacer algo del estilo, que "hay que buscarle un sentido que no se ve muy fácilmente", motivos personales de poca originalidad y falta de autoconfianza o dificultades para entender lo que se estaba haciendo.

Figura 14. P. 3. ¿Te resultó fácil encontrar una solución para la baldosa estropeada?

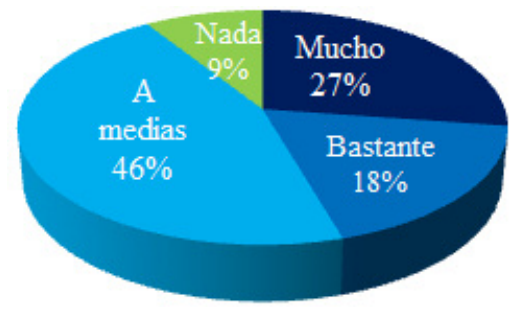

Figura 15. Resultados por edad P.3.

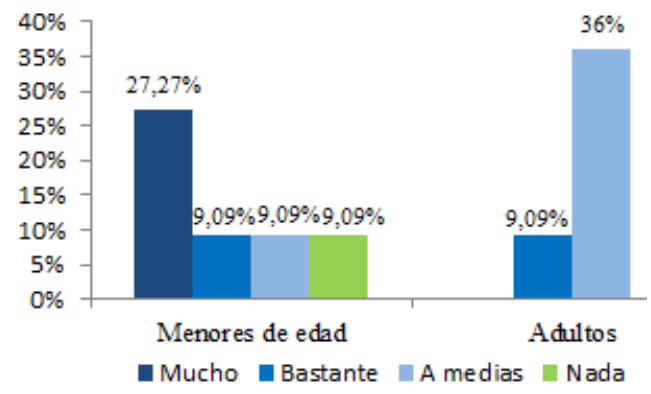


A pesar de la dificultad percibida por los adultos, el $60 \%$ de ellos, finalmente, consiguió ver bastante potencialidad a la baldosa rota, con reflexiones acerca del aprovechamiento de los desperfectos para sacar otra cosa. Los menores ven mucha potencialidad en dicha baldosa en un $50 \%$ de los casos, declarando que "de lo roto se podían sacar buenas cosas" (ver Figura 16 y Figura 17).

Figura 16. P.4. ¿Conseguiste ver potencialidades a la baldosa rota? -

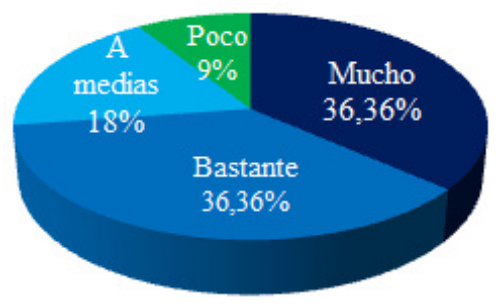

Figura 17. Resultados por edad P.4.

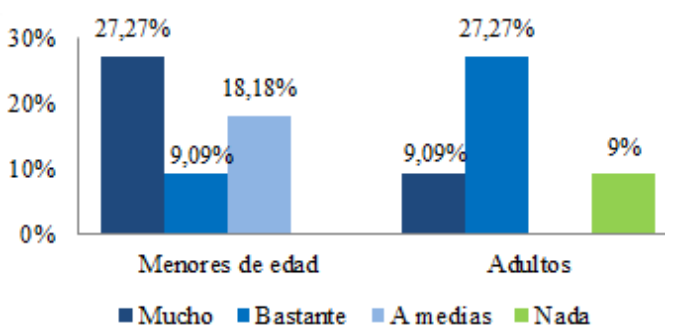

A continuación, se analizan los resultados de las preguntas acerca de la reflexión personal y grupal ocurrida durante el desarrollo de la actividad. Cabe destacar que estas tres preguntas son las menos contestadas, sin respuesta del 36,36\% de los participantes en el caso de la P.7.

Respecto a las similitudes entre problemas medioambientales, sociales y personales, se encuentra una mayor dificultad para relacionarlos por parte de los menores, de los cuales un 75\% las encuentran "a medias" y un $25 \%$, "bastante", relacionando la guerra con la quema de bosques y la contaminación. Por otro lado, el $60 \%$ de los adultos expresa encontrar bastante similitud, porque "todo influye en la persona" y "todo está relacionado en la vida" (ver Figuras 18 y 19).

Figura 18. P.5. ¿Encontraste similitudes entre los problemas ambientales, sociales y personales?

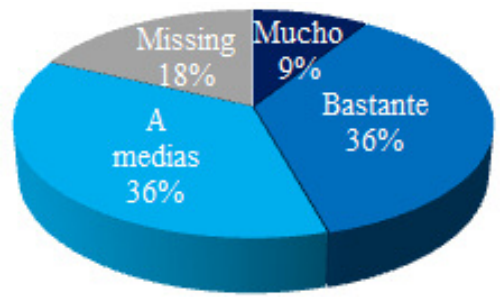


Figura 19. Resultados por edad P.5.

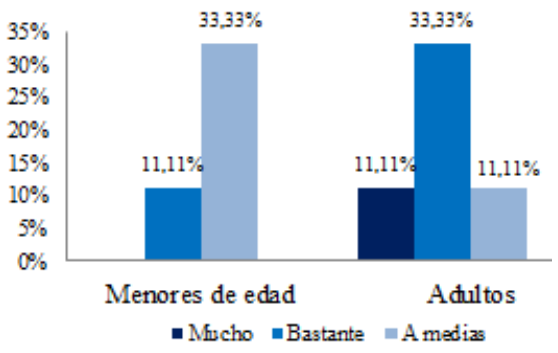

En cuanto al cambio de opinión (ver Figuras 20,21, 22 y 23), encontramos un 37,5\% de personas que han cambiado mucho alguna opinión personal, todas adultas y con declaraciones acerca del cambio de perspectiva y de la mayor motivación que aporta la creatividad y el arte a la hora de trabajar la concienciación medioambiental; quedando el grupo de menores repartido entre "poco", con un 12,5\%, "a medias", con un $25 \%$ y "nada", con otro $25 \%$, de los cuales alguno expresa haber cambiado de opinión sobre la necesidad de "cambiar el medio ambiente" o "lo importante de actuar de inmediato para resolver los problemas".

Figura 20. P.6. ¿Has cambiado de opinión o formado una nueva opinión sobre algo?

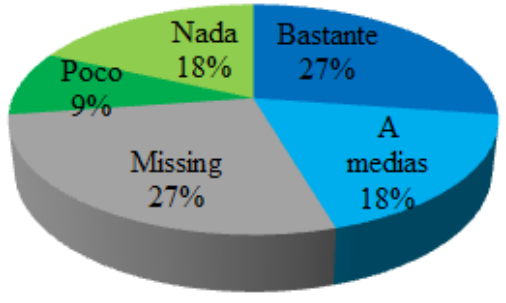

Figura 21. Resultados por edad P.6.

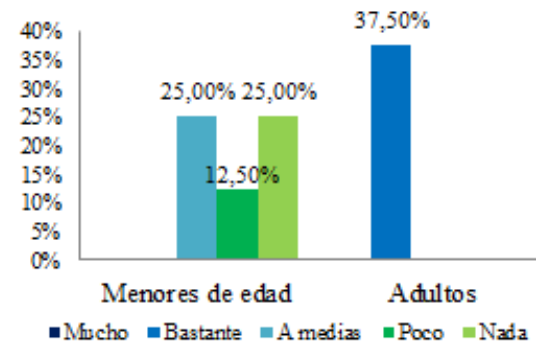


Figura 22. P.7. ¿Has contribuido al cambio de opinión de alguno de los compañeros?

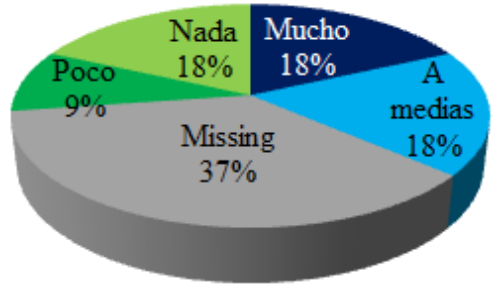

Figura 23. Resultados por edad P.7.

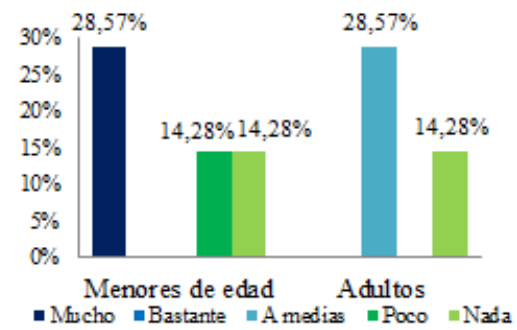

Por último, se analizan los resultados de la pregunta libre (P.L.), la cual anima a escribir sobre expectativas, emociones, pensamientos, reflexiones y/o satisfacción respecto a la actividad.

En primer lugar, se encuentra que la participación en esta última pregunta corresponde al $54,55 \%$ de participantes, todas mujeres tanto menores como adultas (ver Figuras 24, 25 y 26).

Figura 24. Participación en la P.L.

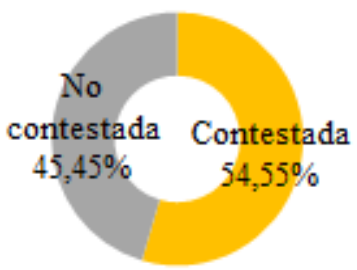

Figura 25. Participación por género en la P.L.

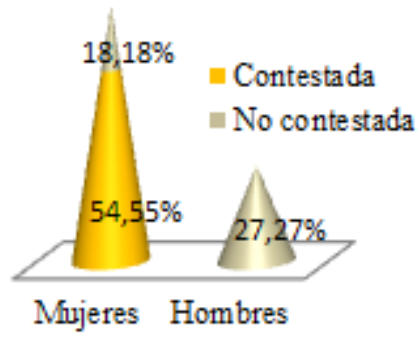


Figura 26. Participación por edad en la P.L.

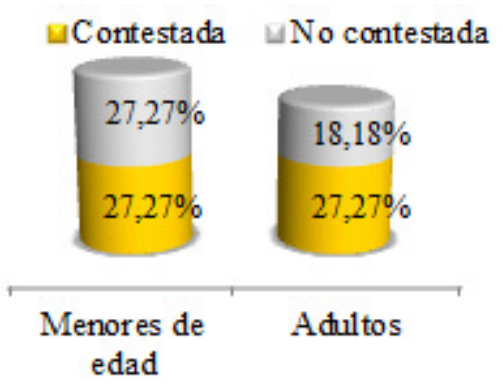

En segundo lugar, esta pregunta sirve para identificar los aspectos de la actividad a los que le han otorgado más valor. El más recurrente es el buen clima grupal y convivencia, con declaraciones como "se está tranquilo, he estado muy relajada", "me he sentido cómoda, atendida y satisfecha", "compartir la actividad con adolescentes le da frescura y ves espontaneidad e implicación", "me resulta interesante estar haciendo algo con el barro con otras personas de diferente edad" o "me ha gustado venir porque me he sentido bien con todos".

También la fase de reconstrucción de la baldosa ha sido destacada en algunas ocasiones expresando gran satisfacción por la inspiración que les ha proporcionado.

Otro de los aspectos destacados es la novedad, con afirmaciones como "siempre es un placer aprender algo nuevo y más cuando tiene un sentido".

Además, se encuentran declaraciones como "me he sentido bien y lo he hecho con ganas". Incluso se aprecia agradecimiento por parte de uno de los menores, ya que expresa que le gusta que hayamos contado con ella.

El único aspecto negativo nombrado, en una única ocasión, ha sido el hecho de mancharse con el barro; a pesar de lo cual, expresando una gran satisfacción general.

Por último, el profesor de barro ofrece una reflexión acerca de "la imposibilidad de ir por la calle y encontrar un adolescente con una persona de sesenta años, es impensable, los valores son distintos, ellos son unas personas muy establecidas; sin embargo, hay un momento de magia en el que todos estuvieron de alguna manera en sintonía, creando, hablando de algo como era el medio ambiente; la necesidad de lo que les rodea, de cambiarlo, lo dijeron a través del barro".

\section{CONCLUSIONES}

El mural tiene un valor estético, el barro, los barnices, los relieves, las formas, no de igual calidad técnica, pues está elaborado por personas muy diferentes y de diferentes capacidades artísticas y sociales, con lo cual habría sido fácil que se generaran recelos, envidia, competitividad... Sin embargo, el aspecto más destacado en las encuestas fue el buen clima grupal, lo cual también forma parte del objetivo, pues no sólo están debilitadas las relaciones con la naturaleza sino entre las propias personas y una forma de lograr el cuidado del planeta, que es nuestro hogar y el de otros, es conociendo y apreciando a los demás. Este mural, en cierto modo, apuesta por una educación ambiental a partir de las relaciones humanas. En este aspecto, se puede concluir la capacidad de unión, entendimiento, convivencia y motivación de las actividades de desarrollo creativo.

Se remarca también el papel como herramienta liberadora, pues su desarrollo genera en las personas la consciencia de la infinidad de posibles perspectivas ante algo, de modo que es útil a la hora de eliminar prejuicios y abrir mentes respecto a temas sociales controvertidos donde las actitudes intolerantes limitan libertades. Normalizar el sufrimiento por ser diferente no es posible en una sociedad cada vez más diversa, se ha de dar la vuelta y ver como normal, bueno y deseable lo diferente, lo cual Ileva un trabajo de desarrollo creativo y pensamiento divergente. 
Además, se destaca la necesidad de formación del profesional a cargo de una actividad artística, de modo que permita la consecución de un objetivo más allá de la propia creación estética, un objetivo social, educativo, terapéutico.

En todas las profesiones prácticas el cómo actuar es una de las grandes incógnitas, pues no hay una receta mágica, sino diversidad de situaciones y de posibles respuestas a ellas. Es en este sentido en el que el arte nos abre puertas y creemos que la más valiosa respuesta encontrada tras este trabajo es a esta pregunta (a cómo intervenir) y la respuesta es Arte, el medio artístico y de desarrollo creativo construye puentes/redes en y entre las personas.

Por último, caben destacar posibles mejoras, como la ampliación o integración de la actividad piloto dentro de un proyecto más amplio, evitando que quede en una actividad aislada, sino que pudiera estar incluida en un programa ambiental o de desarrollo y crecimiento personal en el que participaran otros profesionales desde otros campos, perspectivas o actividades, manteniendo el hilo conductor y permitiendo una mayor profundización en él.

\section{REFERENCIAS BIBLIOGRÁFICAS}

Amabile, T. (1989). Growing Up Creative. New York: The Creative Education.

Calvo Roy, S. y González de la Campa, M. (Coord.) (s.f.). Libro blanco de la educación ambiental en España en pocas palabras. Madrid: Ministerio de Medio Ambiente-Secretaría General de Medio Ambiente. Recuperado de: https://www.miteco.gob.es/es/ceneam/recursos/documentos/libro_blanco.aspx

Edelvives (2019). Croqueta. Recuperado de: https://www.edelvives.com/pr/edelvives/croqueta/

Fundación Tormes (2019, 14 de febrero). Conferencia educativa Educación Socioambiental. Almenara de Tormes, Salamanca.

Gimeno-Doñate, Mㅡㄹ M. (2018). La necesidad de nuevas herramientas para la regulación del proceso de aprendizaje y la mejora del rendimiento académico. Edupsykhe, 15, (2), 82-112. Recuperado de: https://journals.ucjc.edu/EDU/article/view/3976/2925

Guillén, J. (2019, 9 de febrero). Curso presencial Neuroeducación en el aula: De la teoría a la práctica. Integratek, Valladolid.

Marina, J.A. y Marina, E. (2013). El aprendizaje de la creatividad. Barcelona: Editorial Planeta.

Martín González, M.T. y Marín Viadel, E. (2006). Axiología y Creatividad. En S. de la Torre y V. Violant (coord. y dir.), Comprender y evaluar la creatividad: Un recurso para mejorar la calidad de la enseñanza. Vol. 1 (pp. 53-60). Málaga, España: Ediciones Aljibe.

Meller, P. (2019). Claves para la educación del futuro: Creatividad y pensamiento crítico. Santiago de Chile: Catalonia.

Menchén, F. (2006). Pensamiento Lateral de Edward De Bono. En S. de la Torre y V. Violant (coord. y dir.), Comprender y evaluar la creatividad: Un recurso para mejorar la calidad de la enseñanza. Vol. 1 (pp. 41-52). Málaga, España: Ediciones Aljibe.

Renault (2019). Feliziudad. Recuperado de: https://feliziudad.renault.es/

Reyes, M. (2018). El abrazo del arte para la sanación mental. El Mundo. Recuperado de: https://www.elmundo.es/baleares/2018/12/07/5c0a273cfc6c8363718b45a8.html

Romo, M. (2007). Epistemología y psicología. Madrid: Ediciones Pirámide.

Romo, M. (1997). Psicología de la creatividad. Madrid: Paidós.

Scuola de Barbiana (2006). Carta a una maestra. Madrid: PPC.

Solar Rodríguez, M.I. (2006). Creatividad en las capacidades múltiples. En S. de la Torre y V. Violant (coord. y dir.), Comprender y evaluar la creatividad: Un recurso para mejorar la calidad de la enseñanza. Vol. 1 (pp. 31-40). Málaga, España: Ediciones Aljibe.

Sternberg, R. (1988). The Nature of Creativity. Cambridge: Cambridge University Press.

Torrance, P. y Myers, R.E. (1976). La enseñanza creativa. Madrid: Santillana. 
Torre, S. de la (2006a). Creatividad comunitaria y social. En S. de la Torre y V. Violant (coord. y dir.), Comprender y evaluar la creatividad: Un recurso para mejorar la calidad de la enseñanza. Vol. 1 (pp. 569-585). Málaga, España: Ediciones Aljibe.

Torre, S. de la (2006b). Teoría interactiva y psicosocial de la creatividad: Una aproximación basada en el pensamiento complejo. En S. de la Torre y V. Violant (coord. y dir.), Comprender y evaluar la creatividad: Un recurso para mejorar la calidad de la enseñanza. Vol. 1 (pp. 123-154). Málaga, España: Ediciones Aljibe.

Trillas, E. (2018). El desafío de la creatividad. Santiago de Compostela: Servizo de Publicacións e Intercambio Científico Campus Vida.

ZOES (2017). Conclusiones del I Encuentro Ibérico de Barrios Creativos. Recuperado de: https://zoes.es/barrios-creativos/ 